\title{
Im Grenzbereich zwischen Kopfschmerzen und Schlaganfall
}

\author{
Migräne oder Schlaganfall? Eine solche Differenzie- \\ rung ist bei den seltenen akuten neurovaskulären \\ Erkrankungen wie posteriores reversibles Enzephalo- \\ pathiesyndrom (PRES) oder reversibles zerebrales Va- \\ sokonstriktionssyndrom (RCVS) nicht möglich. Wann \\ sollte man an diese seltenen Erkrankungen denken?
}

Eine junge Patientin, die bei einer idiopathischen aplastischen Anämie eine Stammzelltransplantation und eine kombinierte Immunsuppression mit Ciclosporin und Sirolimus erhält, klagt am Tag nach der Transplantation über einen plötzlich einsetzenden starken okzipitalen Kopfschmerz mit Blutdruckanstieg. Am folgenden Tag tritt ein tonisch-klonischer Krampfanfall auf. Bei der bildgebenden Diagnostik finden sich ischämische Veränderungen im posterioren Versorgungsgebiet: hypodense Läsionen im CT und hyperintense Läsionen im MRT.

„Dies ist der typische Fall eines posterioren reversiblen Enzephalopathiesyndroms", sagte Dr. David Seiffge, Stroke Center am Universitätsspital in Basel. Nach Absetzen der Immunsuppressiva habe sich sowohl die klinische Symptomatik als auch der MRT-Befund rasch zurückgebildet. Die typische Symptomkonstellation bei PRES ist der akut einsetzende Kopfschmerz (50\%), Sehstörungen (33\%), epileptische Anfälle (60 - $75 \%$ ), Enzephalopathie (50-80\%) und fokalneurologische Defizite (10 - 15\%). Pathogenetisch dürfte eine Diffusionsstörung mit einem vasogenen beziehungsweise zytotoxischen Ödem vorliegen und zwar als Folge des Funktionsverlustes der Blut-Hirn-
Schranke und einer endothelialen Dysfunktion. Auslösende Faktoren sind Autoimmunerkrankungen wie LE, rheumatoide Arthritis, Sklerodermie, M. Crohn und Colitis ulcerosa, Wegener Granulomatose, Immunsuppressiva und Chemotherapeutika (Ciclosporin, Tacrolimus, Sirolimus, Sunitinib, Sorafenib, Bevacizumab), Sepsis, Hypertonie, akutes oder chronisches Nierenversagen und Eklampsie. „Eine spezifische Therapie gibt es nicht. Vorrangig sind das Ausschalten der auslösenden Ursache und symptomatische Maßnahmen wie Antikonvulsiva und Analgetika“, so Seiffge. Die Prognose sei gut, innerhalb von zwei bis acht Tagen bildet sich die Symptomatik zurück, nur etwa $15 \%$ haben nach einem Jahr noch epileptische Anfälle.

\section{RCVS: ein ähnliches Krankheitsbild}

Beim RCVS werden die rezidivierenden Kopfschmerzattacken durch Vasospasmen verursacht, angiografisch finden sich perlschnurartige Veränderungen an den Gefäßen. Das Krankheitsbild kann spontan auftreten, aber auch eine Schwangerschaft oder vasoaktive Medikamente wie SSRI, Cannabis oder Hormone können der Auslöser sein. Oft finden sich Begleitsymptome wie Nausea, Erbrechen und Photophobie. Differentialdiagnostisch muss immer eine Arteriitis diskutiert werden. Meist bilden sich die Symptome innerhalb von ein bis drei Monaten zurück, selten können eine Blutung oder ein ischämischer Insult auftreten.

Dr. med. Peter Stiefelhagen, Hachenburg

\section{Kryptogener Insult - steckt asymptomatisches VHF dahinter?}

Neuere Studien zeigen, dass zumindest bei einem Teil der Patienten mit einem kryptogenen Insult ein asymptomatisches Vorhofflimmern (VHF) die eigentliche Ursache darstellt. Für solche Patienten besteht dann die Indikation für eine orale Antikoagulation.

In Deutschland leiden 1,6 Millionen Patienten an VHF, eine Million von ihnen weiß es nicht. Unabhängig davon, ob VHF mit Symptomen oder asymptomatisch verläuft, das Risiko für einen Insult ist immer um das Vier- bis Fünffache erhöht und 20 - $30 \%$ aller Schlaganfälle sind kardioembolisch bedingt. „Doch jeder vierte bis fünfte ischämische Insult ist kryptogen, man findet keine eindeutige Ursache“, sagte Professor Joachim Röther, Neurologische Universitätsklinik Hamburg. Dieser Anteil variiere in Abhängigkeit von der Intensität der Diagnostik. Je umfangreicher die Diagnostik, umso seltener sei der Insult kryptogen. Angesichts der Häufigkeit des VHF und der Tatsache, dass diese Rhythmusstörung oft asymptomatisch bleibt, stellt sich die Frage, ob es sich zumindest bei einem Teil der Patienten mit vermeintlich kryptogenem Insult um ein kardioembolisches Ereignis als Folge eines bisher unbekannten VHF handeln könnte.
„Untersuchungen haben gezeigt, dass man mit einem 24-Stunden-EKG nur bei knapp $3 \%$ dieser Patienten ein VHF findet", so Röther. Der Anteil steige aber auf fast $25 \%$, wenn man den Herzschlag mittels Loop-Recorder über vier Wochen aufzeichne. In der ASSERT-Studie wurde bei ICD-Patienten innerhalb von drei Monaten bei 10,1\% zumindest eine asymptomatische VHF-Episode dokumentiert und bei diesen Patienten war das Risiko für Schlaganfall oder systemische Embolie um das 2,5-Fache erhöht. „Doch wir fanden keine zeitliche Korrelation zwischen dem Auftreten der Rhythmusstörung und dem klinischen Ereignis“, so Röther. Dadurch werde die klassische These, dass VHF über eine Stase zu einer Gerinnselbildung und somit zu einem Schlaganfall führe, zerstört. Man müsse diskutieren, dass das VHF selbst kein kausaler Faktor für den Insult sei, sondern nur ein Indikator für eine generelle Emboligenität oder Endothelopathie, die letztendlich für die Thrombusentstehung verantwortlich sei.

\section{Antikoagulation bei Detektion von VHF}

Auch in der CRYSTAL AF-Studie ging man der Frage nach, ob sich hinter dem kryptogenen Insult ein VHF als Ursache verbergen könne. Innerhalb von zwölf Monaten wurde bei kontinuierlicher EKG-Überwachung ein VHF bei 12,4 \%, im Kontroll- 